\title{
Stability and stoichiometry of some binary fluorophore-cyclodextrin complexes
}

\author{
Francesca D’Anna, * Serena Riela, Paolo Lo Meo and Renato Noto* \\ Dipartimento di Chimica Organica 'E. Paternò', Università di Palermo, Viale delle Scienze, Parco d'Orleans II, 90128 Palermo, Italy
}

Received 5 March 2004; revised 8 April 2004; accepted 29 April 2004

\begin{abstract}
The stability and stoichiometric ratio of binary complexes among five fluorophores and $\beta$-cyclodextrin ( $\beta$-CD) or heptakis- $(6-$ amino-6-deoxy)- $\beta$-cyclodextrin (am- $\beta-\mathrm{CD}$ ) were determined by means of fluorescence measurements in borate buffer at $\mathrm{pH}=8.0$ and 9.0 . Structure of both host and guest affected the characteristics of the binary complexes. Pyrene and anthraquinone formed a 1:2 (fluorophore: cyclodextrin) complex with both cyclodextrins. Xanthone formed 1:1 complex with $\beta$-CD and 1:2 complex with am- $\beta$-CD. A more defined behaviour was observed for crysene. In fact, both stoichiometric different complexes were detected with both hosts. Only 1:1 complexes were observed for antracene. The complex stability was affected by the $\mathrm{pH}$ of the solution. MM2 calculations were performed in order to gain information about the forces working on the formation of complexes.

(C) 2004 Elsevier Ltd. All rights reserved.
\end{abstract}

\section{Introduction}

Complexation reactions involving cyclodextrins are highly important in several fields. ${ }^{1}$ These reactions also serve as excellent models for understanding general inclusion phenomena as well as enzyme-substrate interactions. ${ }^{2}$ Recently, we have addressed our interest to the use of cyclodextrin complexes for chiral recognition. ${ }^{3}$ This is one of the main topics that has attracted researchers' attention not only for its important applications in separation science and in medicinal chemistry, but also for its implications in supramolecular catalysis. Chiral recognition by native and modified cyclodextris (CDs) has had and still has a great of importance.

Data collected so far have usually been explained by two different theories: the 'lock-and-key mechanism', ${ }^{4}$ that considers chiral recognition ability as a result of host and guest complementarity in size and in shape; the 'threepoint-rule ${ }^{5}$ that, considers chiral recognition ability in terms of non covalent interactions such as electrostatic interactions, hydrogen bonds, and coordinate bonds.

However, results reported so far suggest that the ability of native and modified cyclodextrins to discriminate between enantiomers of a chiral guest is not very high. On this subject Tabushi et al., ${ }^{6}$ in their pioneering work on chiral recognition, reported that $6^{\mathrm{A}}$-amino- $6^{\mathrm{B}}$-carboxy- $6^{\mathrm{A}}, 6^{\mathrm{B}}$ -

Keywords: Cyclodextrins; Binary complexes; Fluorescence.

* Corresponding authors. Tel.: +39091596919; fax: +39091596825; e-mail address: rnoto@unipa.it deoxy- $\beta$-cyclodextrin has a poor enantioselectivity for enantiomers of tryptophan.

Similarly Kitae and Kano, ${ }^{7}$ studying the binding properties of 6-amino-6-deoxy- $\beta$-cyclodextrin and heptakis-(6-amino6 -deoxy)- $\beta$-cyclodextrin versus $N$-acetylated-Trp, -Leu and -Phe, reported that protonated amino- $\beta$-cyclodextrins bind preferably with L-enantiomers and attributed low enantioselectivity values (1.04-1.54) to small structural differences between the complexes formed by enantiomers.

Good results have been obtained by Marchelli, Rizzarelli et al., ${ }^{8}$ who pointed out the particular affinity of $\mathrm{Cu}(\mathrm{II})-6$ deoxy-6-histamine- $\beta$-cyclodextrin for D-enantiomer of some native $\alpha$-aminoacids. Only recently, Liu et al., ${ }^{9}$ studying the binding properties of some organoselenomodified- $\beta$-cyclodextrins, reported that mono-2-phenylseleno-2-deoxy- $\beta$-cyclodextrin gives a high L-enantioselectivity for the inclusion complexation of leucine (up to 8.4).

Recently, we reported data about the effect of some $\alpha$-amino acids and their corresponding methyl esters on the stability of the binary complex formed by pyrene (Py) in the presence of heptakis-(6-amino-6-deoxy)- $\beta$-cyclodextrin (am- $\beta$ CD). ${ }^{3}$ On that occasion it was pointed out that the binary complex Py/am- $\beta-C D$, having a 1:2 stoichiometric ratio, is a good chiral selector. In fact L-enantioselectivity determinated at $\mathrm{pH}=8.0$, in borate buffer, ranges from 1.2 up to 7.4.

Owing to the nature of the complex formed, this significant chiral recognition ability was thought to be due to the 
extension of the empty volume of the CD cavity that could be differently occupied by enantiomers of the same amino acid.

Our opinion seems to agree with Buvári-Barcza et al., ${ }^{10}$ who observed that the chiral selectivity of different derivatives of $\beta-C D$ changes with the degree of substitution, that in turn can influence the cavity size. It is possible, furthermore, that the stoichiometric ratio $(1: 1$ and/or $1: 2$, fluorophore:CD) of the binary complex and that $(1: 1: 1,1: 2: 1$ or $1: 2: 2$, fluorophore:CD:ternary agent) of the ternary one may be relevant in determining the extent of the chiral recognition.

However, we believe that direct substrate-CD interaction is not comparable with substrate-binary complex interaction. Indeed, the former leads to the best host-guest fit, whereas the latter should consist of an acceptable arrangement of complex.

Therefore, in order to study the importance of size cavity in the chiral discrimination ability of binary complexes, we carried out this preliminary study on the structural characteristics of complexes formed by $\beta$-cyclodextrin and heptakis-(6-amino-6-deoxy)- $\beta$-cyclodextrin in the presence of some suitable different guests (Fig. 1).

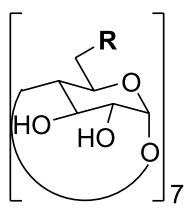

$\mathbf{R}=\mathrm{OH}$

$\mathrm{NH}_{2}$
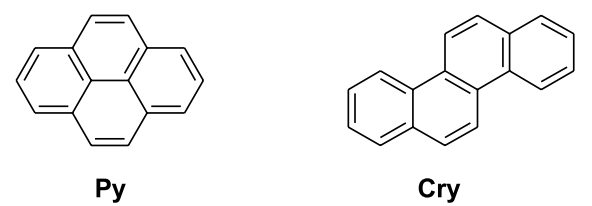<smiles>c1ccc2cc3ccccc3cc2c1</smiles>

An<smiles>O=c1c2ccccc2oc2ccccc12</smiles>

Xan<smiles>O=C1c2ccccc2C(=O)c2ccccc21</smiles>

$\mathrm{Aq}$
Figure 1. Hosts and guests structures.

This investigation was carried out by spectrofluorimetric titration, in borate buffer, at two $\mathrm{pH}$ values (8.0 and 9.0).

Hosts were chosen in order to evaluate the effect that substitution, on going from $\beta-C D$ to am- $\beta-C D$, may exert on complex stability and stoichiometric ratio. These factors could also be influenced by the $\mathrm{pH}$ value, considering that am- $\beta-\mathrm{CD}$, going from $\mathrm{pH}=8.0$ to $\mathrm{pH}=9.0$, passes from its charged form to its neutral form. ${ }^{11}$

Similarly, fluorophore guests were chosen for their different shapes and sizes, that can influence the size of the empty cavity, but also for their different polarities.
In order to have a better knowledge of the forces working on the formation of complexes, their models were elaborated in the gas phase by computational tools.

\section{Results and discussion}

In Table 1 the values of stability constant, as a function of $\mathrm{pH}$ value, and stoichiometric ratios are reported. In any case the stoichiometric ratio was determined by Job's plot ${ }^{12}$ and this result was always confirmed by the Benesi-Hildebrand double-reciprocal plot. ${ }^{13}$

Table 1. Measured binding constants

\begin{tabular}{|c|c|c|c|c|c|c|}
\hline Guest & Host & $\mathrm{pH}$ & Stoich. ratio & $\begin{array}{l}\beta_{2} / 10^{6} \\
\left(\mathrm{M}^{-2}\right)\end{array}$ & $\begin{array}{c}K_{1} \\
\left(\mathrm{M}^{-1}\right)\end{array}$ & $\begin{array}{c}K_{2} \\
\left(\mathrm{M}^{-1}\right)\end{array}$ \\
\hline \multirow[t]{4}{*}{ An } & $\beta-C D$ & 8.0 & $1: 1$ & & 190 & \\
\hline & $\beta-C D$ & 9.0 & $1: 1$ & & 780 & \\
\hline & $a m-\beta-C D$ & 8.0 & $1: 1$ & & 680 & \\
\hline & $a m-\beta-C D$ & 9.0 & $1: 1$ & & 2500 & \\
\hline \multirow[t]{4}{*}{ Cry } & $\beta-C D$ & 8.0 & $1: 1+1: 2$ & & 2800 & 3800 \\
\hline & $\beta-C D$ & 9.0 & $1: 1+1: 2$ & & 2300 & 1560 \\
\hline & $a m-\beta-C D$ & 8.0 & $1: 1+1: 2$ & & 2000 & 2700 \\
\hline & am- $\beta-C D$ & 9.0 & $1: 2$ & 3.4 & & \\
\hline \multirow[t]{4}{*}{ Py } & $\beta-C D$ & 8.0 & $1: 2$ & 7.5 & & \\
\hline & $\beta-C D$ & 9.0 & $1: 2$ & 12.0 & & \\
\hline & $a m-\beta-C D$ & 8.0 & $1: 2$ & 1.7 & & \\
\hline & $a m-\beta-C D$ & 9.0 & $1: 2$ & 4.8 & & \\
\hline \multirow[t]{4}{*}{ Aq } & $\beta-C D$ & 8.0 & $1: 2$ & 2.4 & & \\
\hline & $\beta-C D$ & 9.0 & $1: 2$ & 10.8 & & \\
\hline & $a m-\beta-C D$ & 8.0 & $1: 2$ & 1.4 & & \\
\hline & $A m-\beta-C D$ & 9.0 & $1: 2$ & 2.3 & & \\
\hline \multirow[t]{4}{*}{ Xan } & $\beta-C D$ & 8.0 & $1: 1$ & & 420 & \\
\hline & $\beta-C D$ & 9.0 & $1: 1$ & & 1100 & \\
\hline & $A m-\beta-C D$ & 8.0 & $1: 2$ & 3.7 & & \\
\hline & $A m-\beta-C D$ & 9.0 & $1: 2$ & 4.5 & & \\
\hline
\end{tabular}

All stability constants were reproducible within $10 \%$.

As can be seen from the data reported in Table 1, in many cases the complexation of fluorophore to $\beta-C D$ or to am- $\beta$ $\mathrm{CD}$ can be described by sequential complexation of cyclodextrin molecules (Eqs. 1 and 2): ${ }^{14}$

$\mathrm{S}+\mathrm{CD} \stackrel{K_{1}}{\rightleftarrows} \mathrm{SCD}$

$\mathrm{SCD}+\mathrm{CD} \stackrel{K_{2}}{\rightleftarrows} \mathrm{S}(\mathrm{CD})_{2}$

The overall stability constant will be given by Eq. 3:

$\beta_{2}=K_{1} K_{2}=\left[\mathrm{S}(\mathrm{CD})_{2}\right] /[\mathrm{S}][\mathrm{CD}]^{2}$

If $[\mathrm{CD}] \gg[\mathrm{S}]$ and if the complex having stoichiometric ratio $1: 2$ is predominant, the change of fluorescence intensity as function of CD concentration will be given by Eq. 4:

$\Delta I=\Delta \alpha \beta_{2} \mathrm{~S}_{\mathrm{t}}[\mathrm{CD}]_{0}^{2} /\left(1+\beta_{2}[\mathrm{CD}]_{0}^{2}\right)$

where $\Delta \alpha$ is the difference of emission quantum yields of free and complexed substrate, $\mathrm{S}_{\mathrm{t}}$ and $\mathrm{CD}_{0}$ are the total concentration of substrate and cyclodextrin, respectively.

In the presence of $\beta-\mathrm{CD}$ at $\mathrm{pH}=8.0$ and $\mathrm{pH}=9.0$ or am- $\beta$ $\mathrm{CD}$ at $\mathrm{pH}=8.0$, fluorescence spectra of Cry showed a particular trend. In fact, at a given wavelength, fluorescent intensity firstly increases with $\mathrm{CD}$ concentration, then decreases. In these cases, we have supposed that the two 
different complexes (1:1 and 1:2) were present at comparable concentrations and we analysed experimental data using Eq. 5:

$$
\begin{aligned}
\Delta I= & \left(\mathrm{S}_{\mathrm{t}} K_{1} \Delta(1)[\mathrm{CD}]_{0}+\mathrm{S}_{\mathrm{t}} K_{1} K_{2} \Delta \alpha(2)[\mathrm{CD}]_{0}^{2}\right) / \\
& \left(1+K_{1}[\mathrm{CD}]_{0}+K_{1} K_{2}[\mathrm{CD}]_{0}^{2}\right)
\end{aligned}
$$

where $\Delta \alpha(1)$ and $\Delta \alpha(2)$ are, respectively, the difference of emission quantum yields of free and complexed substrate from 1:1 and 1:2 complexes. Previously, studying the complex formation between $\alpha-\mathrm{CD}$ and para-nitrosubstituted anilines via uv-vis spectroscopy, we observed that the absorbance maximum firstly increases then passes through a maximum and finally decreases on increasing the host concentration. This trend was explained by admitting that two different complexes, having 1:1 and 1:2 stoichiometric ratios, were formed. ${ }^{15}$

All substrates used in this work have shown a good sensitivity to microenvironmental changes. In fact, in all cases considered, we have detected significant changes of fluorescent intensity when the CD concentration increased. In particular all fluorescent probes, except for the Xan, showed a higher fluorescent intensity when they were included in CD cavity.

In the presence of Xan, in both cases, that is, in the presence of $\beta-C D$ and am- $\beta-C D$, fluorescent intensity decreases when the cyclodextrin concentration increases. This result agrees with changes observed in fluorescent intensity by addition of a solvent less polar than water, such as 1,4dioxane, to an aqueous solution of the ketone. ${ }^{16}$

The characteristics of the binary complex fluorophore:CD are obviously affected by different factors. Thus it is really important to consider the different structures of the hosts used.

Indeed, it is common knowledge that substitution of hydroxy groups on the primary rim of the $\beta-C D$ can significantly modify its binding properties, ${ }^{17}$ expecially in the presence of substituents, such as amino groups that, as in this case, change their charge when the $\mathrm{pH}$ value increases. Furthermore, it is important to realize that, the change of electrostatic charge on the am- $\beta-C D$ could have significant consequences on the geometric arrangement of the host. Then $\mathrm{pH}$ variation can be important in determining both stability and stoichiometric ratio of complex.

On the other hand, the guest structure, with its different polarity or hydrophobicity could also affect the characteristics of the system.

\subsection{Host structure}

Data reported in Table 1 show that, when it is possible to compare complexes formed by the two different hosts, by virtue of the same stoichiometric ratio, the native $\beta-C D$ seems to be a better ( $2-3$ times) ligand than am- $\beta-C D$.

This result agrees with Kano's hypothesis ${ }^{18}$ that attributes the lower binding ability of the am- $\beta-C D$, in its partially charged form, to the occurrence of a distorted structure, owing to electrostatic repulsion among charged groups. Furthermore, it should be considered that when amino groups are protonated, they are able to hamper the cavity desolvation process that has always been considered to be one of the essential steps to promote inclusion complex formation. ${ }^{19}$

The binding ability of both hosts is influenced by $\mathrm{pH}$ changes and, independent of the guest considered, they form less stable complexes at $\mathrm{pH}=8.0$ than at $\mathrm{pH}=9.0$.

Presumably the increasing base concentration could break the network of hydrogen bonds on the secondary rim allowing a best fit substrate-cyclodextrin complex.

Furthermore, in general, the increase of complex stability, with increasing $\mathrm{pH}$ value, is higher for $\beta-\mathrm{CD}$ than for am- $\beta$ $\mathrm{CD}$. This result, appears anomalous, considering the extent of charge variation on the am- $\beta-\mathrm{CD}$ at increasing $\mathrm{pH}$ values, can be explained by considering characteristics of buffer used to carry out measurements.

On this topic, it has recently been reported that the stability of host-guest complexes, formed by charged cyclodextrin, can be influenced by the charge of the buffer used. ${ }^{20}$ Under this light, in our opinion, we may presume that the borate anion, is able to partially compensate the positive charge on the am- $\beta-C D$, with an overall decrease of its unfavourable effect, at $\mathrm{pH}=8.0$.

Also the stoichiometric ratio seems to be influenced by the binding ability of the am- $\beta-C D$. Probably the am- $\beta-C D$, owing to geometric modifications of the cavity and to strong solvation, includes the guest less deeply in its cavity. This could explain why in the presence of both Cry and Xan, on going from the $\beta-C D$ to the am- $\beta-C D$, formation of $1: 2$ complexes, becomes favoured.

\subsection{Guest structure}

The guests studied have different polarity and hydrophobicity. In particular hydrophobicity increases going from An to Py or Cry, on increasing the number of fused aromatic rings.

Guests having three fused rings (An, Xan and Aq) differ for characteristics of their central ring. This is hydrophobic for An, moderately hydrophilic and symmetric for Aq, more hydrophilic and unsymmetric for Xan.

Data reported in Table 1 show that these structural characteristics are able to influence both the stability and stoichiometric ratio of complexes. On this subject, whereas An forms, both in the presence of $\beta-C D$ and am- $\beta-C D$, complexes having a 1:1 stoichiometric ratio, more hydrophobic guests (Py and Cry) show a marked trend to form complexes having a 1:2 stoichiometric ratio.

However, comparison among these guests also shows that molecular shape is important. Indeed, the non linear structure of Cry seems to hamper the formation of species having a 1:2 stoichiometric ratio. This result could explain 
why in the presence of this guest, having four aromatic rings as $\mathbf{P y}$, both complexes (1:1 and 1:2) are present in comparable amounts.

Complex stability for guests of similar hydrophobicity changes with their shape. Indeed, considering 1:2 complexes (Cry/am- $\beta-\mathrm{CD}$ and Py/am- $\beta-\mathrm{CD}$ at $\mathrm{pH}=9.0)$ the more symmetric molecule forms a more stable complex.

Among guests having three fused rings, complexes having a $1: 2$ stoichiometric ratio begin to predominate going from An to Aq.

In the presence of $\beta-C D$, Xan forms a 1:1 complex. The same result was previously found by Bohne et al. ${ }^{21}$ Probably, in this case, a favourable dipole-dipole interaction is operative. Indeed, the Xan molecule should be included in a such manner that its $\mathrm{C}_{2}$ symmetry axis is not parallel to the secondary rim of $\beta$-CD. The endocyclic oxygen atom of the Xan molecule should be located near to the rim whereas the carbonyl group is directed towards the bulk of solution. This could justify the preference for the 1:1 complex with $\beta$-CD. This arrangement allows hydrogen bond formation between the secondary hydroxy groups of $\beta-C D$ and oxygen atom of the guest. In our opinion, this additional interaction can explain why the complex formed by Xan is more stable than that formed by more hydrophobic An.

Aq forms complexes having a 1:2 stoichiometric ratio, both in the presence of $\beta-C D$ and am- $\beta-C D$. Recently Dong et al., ${ }^{22}$ studying inclusion of some pharmaceutically related molecules, reported that $\mathbf{A q}$, in the presence of $\beta-C D$ forms a 1:1 complex, where only the hydrophobic part of the guest penetrates in host cavity. This different result could be a consequence of the different host/guest ratios used in the two cases.

However, Aq/am- $\beta-C D$ complexes are less stable than the Xan/am- $\beta-C D$ ones. Probably, in this case, notwithstanding the same stoichiometric ratio, higher stability could be due to hydrogen bonds that Xan can form with secondary rim of am- $\beta-C D$.

\subsection{Computational models}

Further insights were achieved by means of suitable computational tools. Models of the complexes in the gas phase were elaborated and subjected to full geometry optimisation, by means of a MM2/QD 23 molecular mechanics method (see Section 4). Computational data, reported in Table 2, allowed us to calculate the energy variations $\Delta E_{\mathrm{r}(1: 1)}$ and $\Delta E_{\mathrm{r}(1: 2)}$ associated to the formation of the $1: 1$ and 1:2 complexes respectively. Noticeably, the am- $\beta-C D$ was considered only in its neutral form (calculations in the gas phase on charged species do not allow reliable enough predictions). ${ }^{23}$

Data reported in Table 2 show that complexes having a 1:2 stoichiometric ratio are always favoured. This could be the result of increasing of hydrophobic interactions.

In the gas phase and in the presence of am- $\beta-C D, \mathbf{X a n}, \mathbf{P y}$ and Aq show a higher tendency to form 1:2 complexes. This result perfectly agrees with that obtained in buffer solution at $\mathrm{pH}=9.0$.

However, in order to perform a comparison with our experimental data in solution, $\Delta E_{\mathrm{r}(1: 2)}$ data are clearly overestimated. This could be due, in our opinion, to the mutual interaction between the two host hydroxylated rims.

The latter energy contribution, $\Delta E_{2 \mathrm{~h}}$, formally related to the ideal process (Eq. 6):

$2 \mathrm{CD} \rightleftarrows(\mathrm{CD})_{2}$

can be easily calculated.

Nevertheless, we may reasonably presume that in solution, owing to the solvation of the rims, this contribution, should be less relevant.

As can be seen by comparing columns 5 and 8 of Table 2, MM2 calculations foresee only for the Py and Cry with $\beta$-CD a higher stability of 1:2 complexes with respect to $1: 1$ ones. For all other fluorophores the 1:1 complexes are calculated to be as stable as, or even, more stable than the

Table 2. Calculated (MM2) binding energies (kcal/mol)

\begin{tabular}{|c|c|c|c|c|c|c|c|c|c|}
\hline Guest & Host & $E_{\text {st/guest }}{ }^{\mathrm{a}}$ & $E_{\mathrm{st} / \mathrm{cplx}(1: 1)}^{\mathrm{b}}$ & $E_{\mathrm{st} / \mathrm{cplx}(1: 2)^{\mathrm{c}}}$ & $E_{\mathrm{st} / 2 \mathrm{CD}}{ }^{\mathrm{d}}$ & $\Delta E_{\mathrm{r}(1: 1)}{ }^{\mathrm{e}}$ & $\Delta E_{\mathrm{r}(1: .2)^{\mathrm{f}}}$ & $\Delta E_{\mathrm{r} 2 \mathrm{~h}}^{\mathrm{g}}$ & $\Delta E_{\mathrm{r}}^{\mathrm{h}}$ \\
\hline \multirow[t]{2}{*}{ An } & $\beta-C D$ & -17.04 & 38.70 & 70.96 & 158.29 & -35.35 & -58.80 & -23.83 & -34.97 \\
\hline & Am- $\beta-C D$ & & 34.03 & 64.00 & 144.40 & -41.49 & -62.59 & -40.72 & -21.87 \\
\hline Cry & $\mathrm{am}-\beta-\mathrm{CD}$ & & 25.72 & 54.00 & 142.54 & -49.44 & -64.28 & -42.58 & -21.7 \\
\hline \multirow[t]{2}{*}{$\mathbf{P y}$} & $\beta-C D$ & -21.37 & 35.44 & 61.57 & 160.89 & -34.25 & -64.23 & -21.23 & -42.98 \\
\hline & $\mathrm{am}-\beta-\mathrm{CD}$ & & 39.92 & 54.47 & 159.76 & -31.27 & -76.51 & -25.36 & -51.15 \\
\hline \multirow[t]{2}{*}{ Aq } & $\beta-C D$ & 8.05 & 56.34 & 95.35 & 163.12 & -42.77 & -52.05 & -19.00 & -33.05 \\
\hline & $a m-\beta-C D$ & & 62.75 & 86.22 & 153.23 & -37.86 & -69.09 & -31.89 & -37.2 \\
\hline \multirow[t]{2}{*}{ Xan } & $\beta-C D$ & 3.89 & 55.14 & 89.60 & 147.78 & -39.81 & -56.6 & -34.34 & -22.26 \\
\hline & am- $\beta-C D$ & & 56.09 & 85.69 & 148.75 & -37.00 & 65.96 & -36.67 & -29.29 \\
\hline
\end{tabular}

\footnotetext{
a Steric energy of guest.

b Steric energy of 1:1 complex.

c Steric energy of 1:2 complex.

d Steric energy of two CD molecule in the (1:2) complex deprived of guest.

e Entalphy of reaction: CD+guest $>$ Cplx $(1: 1)$.

f Entalphy of reaction: Cplx $(1: 1)+\mathrm{CD}>\mathrm{Cplx}(1: 2)$.

Entalphy of reaction: $2 \mathrm{CD}>(\mathrm{CD})_{2}$.

h $\Delta E_{\mathrm{r}(1: 2)}-\Delta E_{\mathrm{r} 2 \mathrm{~h}} E_{\text {ster } / \beta-\mathrm{CD}}=91.06 \mathrm{kcal} / \mathrm{mol} . E_{\text {ster } / \mathrm{am}-\beta-\mathrm{CD}}=92.56 \mathrm{kcal} / \mathrm{mol}$.
} 
1:2 complexes. The latter results appear in fairly good agreement with experimental data. However, some discrepancies are still present. This could be due, in our opinion, partly to the neglect of any entropic contribution, partly to the fact that, in the absence of any explicit solvent environment in the calculation, only the 'naked' host-guest interaction is actually taken into account.

\section{Conclusions}

Data collected in the present work have allowed us to characterize some binary complexes fluorophore: cyclodextrins. We hope that these complexes, having significantly different properties, can show different chiral recognition abilities. Moreover, we have confidence that they will allow us to realize which factors determine the high or low ability of a receptor to act as chiral selector.

\section{Experimental}

\subsection{Materials}

The heptakis-(6-amino-6-deoxy)- $\beta$-cyclodextrin was synthesized and purified according to the procedure described in the literature. ${ }^{24}$ The product was dried for $24 \mathrm{~h}$ in a dryer under vacuum over phosphorous pentoxide at $60^{\circ} \mathrm{C}$ and then was stored in the same apparatus at $40{ }^{\circ} \mathrm{C}$.

Py, Xan, An, Cry and Aq (spectrofluorimetric grade) were purchased from Fluka and used without further purification.

Borate buffer solutions $(0.05 \mathrm{M})$ were prepared according to standard procedure, using freshly double-distilled decarbonised water. The actual $\mathrm{pH}$ of the solutions was recorded using a PH M82 Radiometer equipped with a GK2401C combined electrode.

\subsection{Spectrometric measurements}

The solutions of $\beta-\mathrm{CD}$ and am- $\beta-\mathrm{CD}\left(1.4 \times 10^{-3} \mathrm{M}\right)$ were filtered before use by a Millipore $0.45 \mathrm{~mm}$ filter. Guest aqueous solutions were prepared by injecting a guest solution $(\mathrm{MeOH}$ or 1,4 -dioxane $)\left(\approx 10^{-3} \mathrm{M}\right)$ into a buffer solution. Measurement solutions were prepared by adding increasing volumes of $\mathrm{CD}$ to $1 \mathrm{ml}$ of guest solution into a volumetric flask. In these solutions, the concentration of guest, reported in Table 3, was constant, while the concentrations of $\mathrm{CD}$ increased from $1.4 \times 10^{-4} \mathrm{M}$ to $1.3 \times 10^{-3} \mathrm{M}$. All measurement solutions were de-aerated, before use, by Ar for $12 \mathrm{~min}$.

Table 3. Experimental conditions

\begin{tabular}{lccclcc}
\hline Guest & $\begin{array}{c}C_{\text {guest }} \\
(\mathrm{M})\end{array}$ & $\begin{array}{c}\lambda_{\mathrm{ex}} \\
(\mathrm{nm})\end{array}$ & $\begin{array}{c}\Delta \lambda_{\mathrm{em}} \\
(\mathrm{nm})\end{array}$ & Solvent & $\begin{array}{c}\text { Ex. slit } \\
(\mathrm{nm})\end{array}$ & $\begin{array}{c}\text { Em. slit } \\
(\mathrm{nm})\end{array}$ \\
\hline An & $5 \times 10^{-7}$ & 261 & $360-450$ & $\mathrm{MeOH}$ & 3 & 3 \\
Cry & $2 \times 10^{-7}$ & 276 & $350-450$ & $\mathrm{MeOH}$ & 3 & 3 \\
Py & $2 \times 10^{-7}$ & 337 & $360-450$ & $\mathrm{MeOH}$ & 1.5 & 1.5 \\
Aq/ $\beta-\mathrm{CD}$ & $2 \times 10^{-6}$ & 310 & $320-600$ & $\mathrm{Diox}$ & 5 & 5 \\
Aq/am- $\beta-\mathrm{CD}$ & $2 \times 10^{-6}$ & 310 & $320-600$ & $\mathrm{Diox}$ & 3 & 5 \\
Xan & $4 \times 10^{-6}$ & 348 & $360-450$ & $\mathrm{MeOH}$ & 1.5 & 3 \\
& & & & & &
\end{tabular}

Steady-state fluorescence spectra were acquired with a JASCO FP-777W spectrofluorimeter. Excitation, emission slits, excitation wavelength and emission interval are reported in Table 3 .

Every spectrum was averaged over 50 scans. A suitable wavelength was chosen after recording a 'difference spectrum' by comparison to a sample without cyclodextrin and one with the highest CD concentration.

\subsection{Calculations}

MM2 calculations were performed by means of the CS Chem 3D Pro ${ }^{\mathrm{TM}} 5.0$ software package from the Cambridge Soft Corporation. Models of the host and their complexes were elaborated with the aid of the 'Quenched Dynamics' (QD) method outlined by Lipkowitz. ${ }^{25}$ The behaviour of a suitable starting model of the complex at $300 \mathrm{~K}$ is simulated by molecular dynamics for a period of $1000 \mathrm{ps,} \mathrm{in} \mathrm{order} \mathrm{to}$ get a significant picture of the conformational space. Structures are sampled from the obtained 'simulation pool' and allowed to undergo full geometry optimisation by means of a simulated annealing procedure. In this way, only a limited number of energy minima are found. Data reported in Table 2 refer to the absolute minimum found for each complex.

\section{Acknowledgements}

Financial support from the University of Palermo (funds for selected research topics) and Italian MIUR within the National Research Project 'Non aromatic heterocycles in stereocontrolled process' is gratefully acknowledged.

\section{References and notes}

1. Rekharsky, M. V.; Inoue, Y. Chem. Rev. 1998, 98, 1875-1917.

2. Bender, M. L.; Korniyama, M. Cyclodextrin Chemistry. Springer: Berlin, 1978.

3. D’Anna, F.; Riela, S.; Lo Meo, P.; Gruttadauria, M.; Noto, R. Tetrahedron: Asymmetry 2002, 1755-1760.

4. Cramer, F. In The Lock and Key Principle. Behr, J.-P., Ed.; Wiley: Chichester, 1994; pp 1-23.

5. Ogston, A. G. Nature 1948, 162, 963.

6. Tabushy, I.; Kuroda, Y.; Mizutani, T. J. Am. Chem. Soc. 1986, $108,4514-4518$

7. Kitae, T.; Kano, K. Abstracts of 11th Symphosium on Biofuctional Chemistry, Fukuoka, Japan, October 1996.

8. Corradini, R.; Dossena, A.; Impellizzeri, G.; Maccarrone, G.; Marchelli, R.; Rizzarelli, E.; Sartor, G.; Vecchio, G. J. Am. Chem. Soc. 1994, 116, 10267-10274.

9. Liu, Y.; You, C. C.; Zhang, H. Y.; Zhao, Y. L. Eur. J. Org Chem. 2003, 1415-1422.

10. Buvári - Bacza, Á.; Bacza, L. Talanta 1999, 49, 577-585.

11. Hamelin, B.; Jullien, L.; Guillo, F.; Lehn, J. M.; Jardy, A.; De Robertis, L.; Driguez, H. J. Phys. Chem. 1995, 99, $17877-17885$

12. Job, P. Ann. Chim. 1928, 9, 113-203. 
13. Benesi, H. A.; Hildebrand, J. H. J. Am. Chem. Soc. 1949, 71, 2703-2707.

14. Xu, W.; Demas, J. N.; DeGraff, B. A.; Whaley, M. J. Phys. Chem. 1993, 97, 6546-6554.

15. Lo Meo, P.; D’Anna, F.; Riela, S.; Gruttadauria, M.; Noto, R. Org. Biomol. Chem. 2003, 1, 1584-1590.

16. Barra, M.; Bohne, C.; Scaiano, J. C. J. Am. Chem. Soc. 1990, 112, 8075-8079.

17. (a) Eliseev, A. V.; Schneider, H. J. J. Am. Chem. Soc. 1994, 116, 6081-6088. (b) Kean, D. S.; May, B. L.; Clements, P.; Easton, C. J.; Lincoln, S. F. Aust. J. Chem. 1999, 52, 1157-1163. (c) Sandow, M.; Easton, C. J.; Lincoln, S. F. Aust. J. Chem. 1999, 52, 1151-1155.

18. Kitae, T.; Nakayama, T.; Kano, K. J. Chem. Soc., Perkin Trans. 2 1998, 207-212.

19. Tabushi, I.; Kiyosuke, Y.; Sugimoto, T.; Yamamura, K. J. Am. Chem. Soc. 1978, 100, 916-919.
20. Gosh, M.; Zhang, R.; Lower, R. G.; Seto, C. T. J. Org. Chem. 2000, 65, 735-741.

21. Murphy, R. S.; Barros, T. C.; Barnes, J.; Mayer, B.; Marconi, G.; Bohne, C. J. Phys. Chem. A 1999, 103, 137-146.

22. Dong, X.-J.; Nie, M.-Y.; Tong, J.; Li, H.-L. J. Electroanal. Chem. 1998, 448, 61-67.

23. (a) D'Anna, F.; Lo Meo, P.; Riela, S.; Gruttadauria, M.; Noto, R. Tetrahedron 2001, 57, 6823-6827. (b) Lo Meo, P.; D'Anna, F.; Riela, S.; Gruttadauria, M.; Noto, R. Tetrahedron 2002, 58, 6039-6045.

24. Ashton, P. R.; Koniger, R.; Stoddart, J. F. J. Org. Chem. 1996, 61, 903-908.

25. (a) Lipkowitz, K. B. Chem. Rev. 1998, 98, 1829-1873. (b) Kozár, T.; Venanzi, C. A. J. Mol. Struct. 1997, 395-396. (c) Kozár, T.; Venanzi, C. A. J. Mol. Struct. 1997, 451, 468. 